\title{
О РЕЗОНАНСАХ ПОВЕРХНОСТНЫХ ВОЛН ПРИ РАССЕЯНИИ АКУСТИЧЕСКИХ ВОЛНОВЫХ ПАКЕТОВ УПРУГИМИ ЦИЛ ИНДРАМИ
}

\author{
(Представил Н. Алумяэ)
}

\begin{abstract}
Выполнен анализ временной зависимости акустического давления, рассеянного сплошным упругим цилиндром в идеальную сжимаемую жидкость при коротких и длинных падающих волновых пакетах и различных частотах заполнения. Выделены- основные структурные компоненты рассеянного поля и дана их количественная характеристика. Установлена связь между временной и частотной зависимостями. Во временной области показан резонансный характер взаимодействия переизлученных волновых пакетов, порождаемых упругой поверхностной волной типа Рэлея, в случае, когда на длине окружности цилиндра помещается целое число длин упругой поверхностной волны.
\end{abstract}

1. В процесс рассеяния акустических волновых пакетов сплошными упругими телами в жидкости существенный вклад вносят пакеты, порождаемые упругими поверхностными волнами. Последние возникают на критических углах $\theta_{j}$, когда проекция фазовой скорости упругой поверхностной волны $c_{j}{ }^{p h}$ на направление распространения падающего волнового пакета равна скорости звука в окружающей упругое тело жидкости

$$
\theta_{j}=\arcsin \left(v_{j}^{p h}\right)^{-1}, \quad v_{j}^{p h}=c_{j}^{p h} / c_{0} .
$$

Здесь индексом $j$ помечен тип упругой поверхностной волны. Эти волны обегают поверхность упругого тела по часовой стрелке и против нее, непрерывно излучая в жидкость. Если их затухание вследствие излучения не слишком велико, то волны многократно обегают тело и интерферируют между собой, причем интерференция образуется как за счет взаимодействия волн, бегущих во встречных направлениях, так и за счет наложения волн самих на себя. Акустическое давление в некоторой фиксированной точке наблюдения определяется суперпозицией переизлученных, переотраженных, дифрагированных и отраженного волновых пакетов.

В серии работ $\left[{ }^{1-5}\right]$ проведено изучение вклада в рассеянное поле переизлученных волновых пакетов, обусловленных распространением по сплошному упругому цилиндру поверхностных волн. В ходе решения стационарной задачи рассеяния авторы применили к решению в виде ряда по собственным функциям интегральное преобразование Зоммерфельда-Ватсона, провели анализ особенностей подынтегральной функции и воспользовались процедурой полюсов Редже, хорошо разработанной в квантовой теории рассеяния. Этим способом им удалось показать, что когда частота падающей волны совпадает с собственной частотой некоторой поверхностной волны, имеет место резонанс этой волны. 
Иными словами, резонанс наступает, когда на длине окружности цилиндра укладывается целое число длин упругой поверхностной волны. Резонансный характер взаимодействия продемонстрирован авторами путем построения как частотных зависимостей для некоторых поверхностных волн (типа Рэлея, Стонели, шепчущей галереи) в широком диапазоне изменения частот, так и полярных диаграмм направленности.

В данной работе показывается, как резонансный процесс проявляется во временной области.

2. Будем использовать обозначения и результаты статьи [ $\left.{ }^{6}\right]$. Из рис. 1 [6] видно, что кривая частотной зависимости имеет глубокие острые минимумы в диапазоне частот $x=5-25$. С целью уточнения их величины и местоположения мы пересчитали частотную зависимость с мелким шагом $l_{x}=1 / 512$. Оказалось, что по сравнению с прежним расчетом, выполненным с шагом $l_{x}=10 / 256$, координаты минимумов сдвигаются в пределах крупного шага, а величины отдельных минимумов значительно уменьшаются. В частности, для глубокого резкого минимума в окрестности $x \sim 20$ мы получили $|p(20,019)|=0,02675$.

Временные зависимости $p(\tau)$ рассчитывались в предположении, что давление в падающем волновом пакете изменяется по гармоническому закону. Частота $x_{0}$ и длительность $\delta_{0}$ пакета изменялись в широких пределах

$$
\delta_{0}=s \tau_{0}, \quad \tau_{0}=2 \pi / x_{0}, \quad s=1,2,3, \ldots,
$$

где $x_{0}$ - частота заполнения, $\tau_{0}$ - период частоты заполнения, $s$ - число периодов частоты $x_{0}$ в падающем волновом пакете.

Ниже рассмотрены две типичные временные зависимости, рассчитанные при коротком $(s \sim 1)$ и длинном $(s \gg 1)$ падающих волновых пакетах. При частоте $x_{0}=20,019$ зададим параметры $s=6$ и $s=317$ для получения короткого и длинного падающих волновых пакетов. Выбор этих значений достаточно произволен и не влияет на результат. Счет проведен для точки наблюдения $r=10^{4}, \theta=0$ в случае размещения в воде стального цилиндра. К моменту прихода волновых пакетов в выбранную точку наблюдения дальнее волновое поле полностью сформировалось. В силу того, что точка наблюдения находится на оси симметрии рассеянного поля, переизлученные и дифрагированные волновые пакеты, обусловленные волнами, обегающими цилиндр по часовой стрелке и против нее, приходят к одинаковой фазе. Поэтому временная зависимость суммарного давления в этой точке должна быть проще, чем в остальных.

3. Из рис. 1 видно, что одиночный падающий волновой пакет, определенный параметром $s=6\left(\delta_{0}=1,88\right)$, вызывает последовательность пакетов во временной зависимости. Первый из них связан с процессом отражения от наружной поверхности цилиндра; начиная с $\tau \sim 1$ на него накладывается волновой пакет, обусловленный переотражением от тыльной поверхности цилиндра. За отраженным следуют волновые пакеты, в основном порожденные процессом переизлучения. Цифра у стрелок означает число полных оборотов, совершенных упругой поверхностной волной типа Рэлея вокруг цилиндра до излучения в точку наблюдения. Для лучшей обозримости амплитуды второго и последующих переизлученных волновых пакетов увеличены в десять раз.

Используя некоторые характерные точки кривой временной зависимости в двух последовательно переизлученных волновых пакетах, можно найти относительную фазовую скорость распространения упругой поверхностной волны 


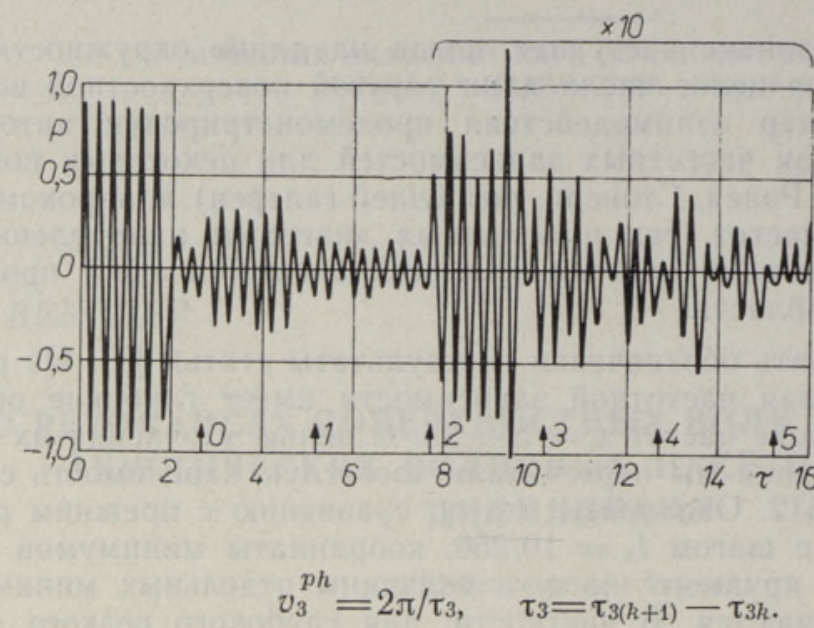

Рис. 1.

$$
v_{3}^{p h}=2 \pi / \tau_{3}, \quad \tau_{3}=\tau_{3(k+1)}-\tau_{3 k} .
$$

Здесь индексом 3 помечены величины, относящиеся к волне типа Рэлея; $\tau_{3 k}$ - время прихода в точку наблюдения некоторой характерной метки, например, начала пакета, в $k$-м переизлученном волновом пакете.

По амплитудам последовательно переизлученных волновых пакетов легко вычислить коэффициент затухания амплитуды поверхностной волны за один оборот пути $\eta_{3}$ и значение амплитуды упругой поверхностной волны $A_{3}$. в момент ее возникновения на критическом угле возбуждения $\theta_{3}$

$$
\begin{gathered}
\exp \left(-2 \pi \eta_{3}\right)=A_{3(k+1)} / A_{3 k}, \quad \theta_{3}=\arcsin \left(v_{3}^{p h}\right)^{-1}, \\
A_{3^{*}}=(1 / 2) A_{30} \exp \left[2\left(\pi-\theta_{3}\right) \eta_{3}\right],
\end{gathered}
$$

где $A_{3 k}-$ величина амплитуды $k$-го переизлученного волнового пакета, вызванного упругой поверхностной волной типа Рэлея.

При выбранных параметрах имеем

$$
\begin{array}{rlrlrl}
\tau_{30} & =2,65, & \tau_{31} & =5,16, & v_{3}^{p h}=2,50, & A_{30}=0,318, \\
A_{33} & =0,036, \quad \theta_{3}=0,412, & \eta_{3}=0,115, & A_{3^{*}}=0,298 .
\end{array}
$$

Отметим, что параметры $\eta_{3}$ и $A_{3}$. определены не очень точно. Это связано не с погрешностью вычисления временной зависимости, а с используемой процедурой нахождения параметров. Фактически волновые пакеты, следующие за отраженным, образуются за счет суперпозиции переизлученных и переотраженных волновых пакетов. Это проявляется в том, что в пределах одного пакета имеет место значительный разброс величин экстремумов, в результате чего огибающие экстремумов соседних волновых пакетов существенно различаются по форме. Независимо проведенный расчет рассеяния волнового пакета на эквивалентном упругому жидком цилиндре (отличие от упругого состоит лишь в том, что не возбуждаются поперечные волны) показал, что процесс переотражения не заканчивается к началу процесса переизлучения. В предлагаемой же процедуре имеется в виду, что вклад переотраженных волновых пакетов невелик и амплитуды следующих за отраженным волновых пакетов определяются исключительно процессом переизлучения. Если провести обработку временной зависимости и выделить в ней отдельные волновые пакеты, т. е. отделить процесс переизлучения от процесса переотражения, то параметры переизлученных волновых пакетов можно будет найти точнее. 

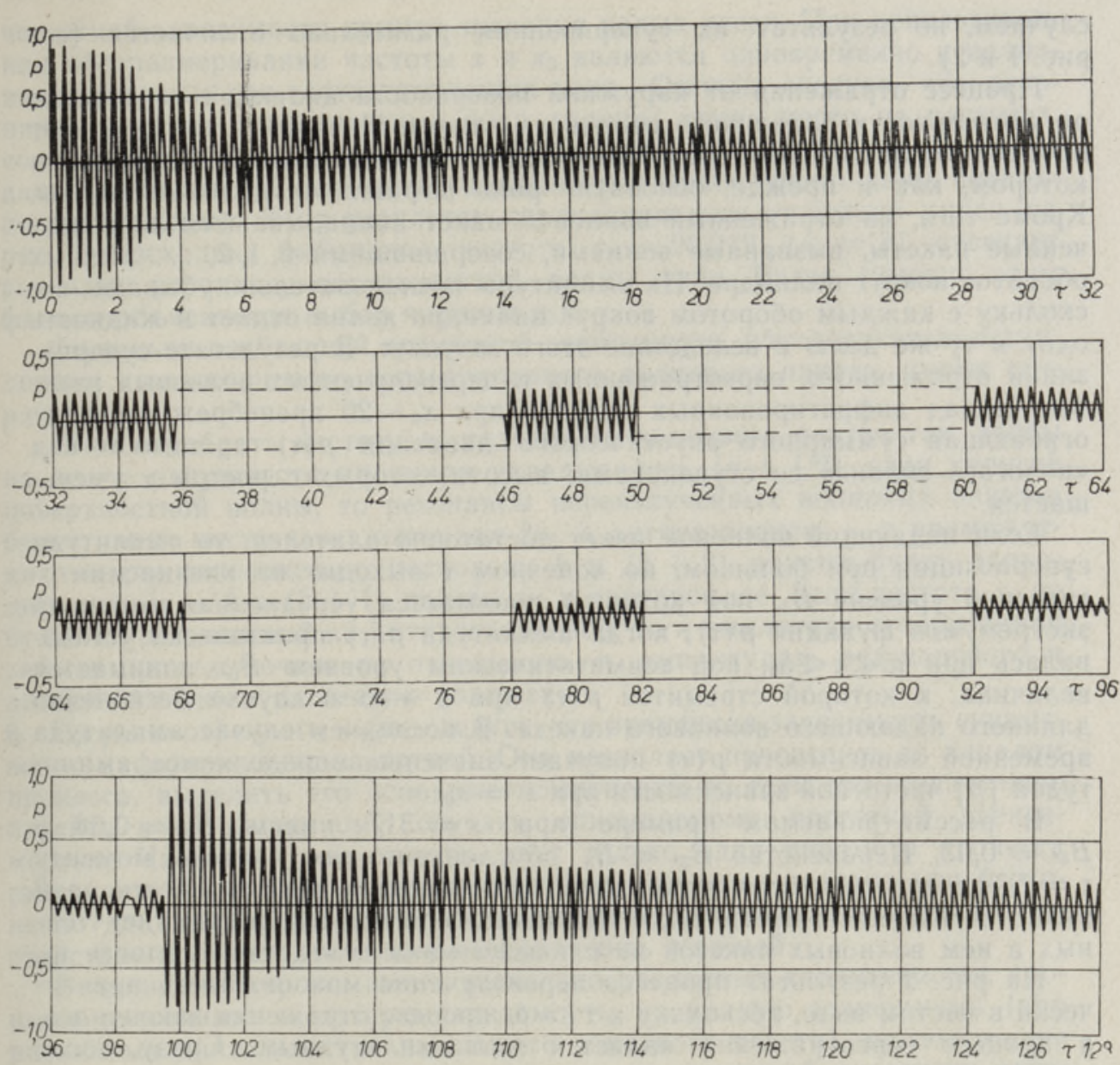

Рис. 2.

По частотной зависимости (см. рис. $1\left[{ }^{6}\right]$ ) найдем значение относительной групповой скорости поверхностной волны типа Рэлея

$$
v_{3}^{g r} \equiv c_{3}^{g r} / c_{0}=2,50 .
$$

Равенство групповой и фазовой скоростей указывает на то, что поверхностная волна распространяется без дисперсии.

При $s=6$ и $\delta_{0}<\tau_{3}$ переизлученные волновые пакеты приходят порознь, не накладываясь друг на друга. При увеличении длительности падающего волнового пакета начиная с $\delta_{0}>\tau_{3}$ переизлученные волновые пакеты частично перекрывают друг друга, поскольку начало пакета индекса $(k+1)$ ложится на конец пакета индекса $k$. C дальнейшим увеличением $\delta_{0}$ наступает такая ситуация, когда начало пакета индекса $(k+2)$ ложится на конец пакета индекса $k$. При очень длинных пакетах, когда $\delta_{0} \gg \tau_{3}$, на каждый переизлученный волновой пакет накладывается достаточно много последующих. Такой случай, рассчитанный при $s=317\left(\delta_{0}=99,49\right)$, показан на рис. 2.

4. Состав волновых пакетов, формирующих рассеянное цилиндром акустическое давление, не изменяется в сравнении с рассмотренным выше 
случаем, но результат их суперпозиции разительно отличается (ср. рис. 1 и 2).

Процесс отражения от наружной поверхности цилиндра не зависит от длительности падающего волнового пакета. Начиная с $\tau \sim 1$ на этот процесс накладывается малоамплитудный процесс переотражения, в котором, как и прежде, основную роль играют продольные волны. Кроме того, на отраженный волновой пакет накладываются переизлученные пакеты, вызванные волнами, совершившими $0,1,2, \ldots$ полных оборотов вокруг цилиндра. Их амплитуды последовательно убывают, поскольку с каждым оборотом вокруг цилиндра волна отдает в жидкость одну и ту же долю и вследствие этого затухает. В результате суперпозиции отраженного, переотраженных и переизлученных волновых пакетов (вклад дифрагированных пакетов при $x_{0} \sim 20$ пренебрежимо мал) огибающая суммарного акустического давления $p(\tau)$ принимает вид «пологого склона» со ступеньками, высота которых с ростом $\tau$ уменьшается.

Если падающий волновой пакет достаточно длителен, то амплитуда суперпозиции при болышом, но конечном $\tau$ выходит на квазиасимптотический уровень $B_{3}$, под которым понимается усредненная величина экстремумов функции $p(\tau)$, когда амплитуда $p(\tau)$ практически установилась при $\tau_{0} \ll \tau<\delta_{0}$; под асимптотическим уровнем $B_{3}$ * понимается величина, к которой стремится $p(\tau)$ при $\tau \rightarrow \infty$ в случае бесконечно длинного падающего волнового пакета. В последнем случае амплитуда временной зависимости $p(\tau)$ получает значение, определяемое амплитудой $|p|$ частотной зависимости при $x=x_{0}$.

В рассматриваемом примере при $s=317$ имеем $B_{3}=0,08$ и $B_{3^{*}}=0,03$. Неравенство $B_{3 *}<B_{3}$ обусловлено тем, что к моменту $\tau=\delta_{0}$ не все переизлученные волновые пакеты успевают внести свой вклад в квазиасимптотическое значение, причем амплитуда неучтенных в нем волновых пакетов не слишком мала вследствие малости $\eta_{3}$.

На рис. 2 результат процесса переизлучения можно видеть практически в чистом виде, поскольку к $\tau>\delta_{0}$ процесс отражения закончился, а процесс переотражения является малоамплитудным. Сразу после $\tau>\delta_{0}$ видна амплитуда суперпозиции всех переизлученных волновых пакетов $C_{3}$, равная 0,822 для рассматриваемого примера; со сдвигом на $\tau_{3}$ из нее последовательно вычитаются амплитуды нулевого, первого, второго и т. д. переизлученных пакетов.

Используя величины амплитуд последовательных ступенек, легко найти значение амплитуды упругой поверхностной волны типа Рэлея в момент ее зарождения на критическом угле и коэффициент затухания; для рассматриваемого примера получим

$$
A_{3^{*}}=0,196, \quad \eta_{3}=0,025 .
$$

Эти величины сильно отличаются от приведенных в (5). Объясняется это тем, что при различных $s$ различен и спектр падающего волнового пакета: при малых $s$ он широкополосный, а при больших $s$ узкополосный. Нам представляется, что параметры $A_{3 *}$ и $\eta_{3}$, найденные в случаях короткого и длинного падающих волновых пакетов, не противоречат друг другу, а скорее наоборот - дополняют друг друга.

5. Если ввести частоту $x_{3}$, естественную для упругой поверхностной волны типа Рэлея

$$
x_{3}=x / v_{3}^{g r},
$$


частотной зависимости примут знанения целых чисел. При использованном обезразмеривании частоты $x$ и $x_{3}$ являются одновременно и волновыми числами для соответствующих волн. Отсюда следует, что резонансы упругих поверхностных-волн должны иметь место на частотах, соответствующих последовательным целым числам (т. е. когда на длине окружности цилиндра укладывается целое число длин поверхностной волны). Этот результат совпадения фаз отчетливо виден на pис. 2, поскольку выбранная частота $x_{0}=20,019 \quad\left(x_{3}=8\right)$ отвечает восьмому резонансу поверхностной волны типа Рэлея (именно, этим фактом объясняется выбор частоты $x_{0}$ ).

Ступеньки огибающей временной зависимости, обусловленные вступлением каждого нового переизлученного волнового пакета, имеют ширину $8 \tau_{0}$.

Если подобрать частоту $x_{0}$ падающего волнового пакета так, чтобы на длине окружности цилиндра укладывалось $(n+1 / 2)$ длин упругой поверхностной волны, то резонансы переизлученных волновых пакетов будут иметь место через интервал $2 \tau_{3}$, а антирезонансы - в промежутках между, резонансами, поскольку $k$ и $(k+2)$ пакеты будут приходить в фазе, а $k$ и $(k+1)$ пакеты - в противофазе. Если частота $x_{0}$ не будет ни резонансной ни антирезонансной, то и амплитуда суперпозиции займет промежуточное по отношению к амплитудам резонансного и антирезонансного рассеяния положение.

6. Из рассмотренного выше следует, что временная зависимость существенно информативнее частотной. Она позволяет проследить за началом процесса, выделить его оснөвные компоненты и, если это желательно, перейти к стационарному случаю, описываемому частотной зависимостью. Частотная зависимость дает только один параметр процесса рассеяния: амплитуду суперпозиции всех волновых пакетов при бесконечно длинном падающем волновом пакете. Тем не менее о некоторых сво́йствах процесса рассеяния можно судить и по ней.

В принципе, хорошо иметь несколько различных характеристик процесса рассеяния: частотную зависимость, временную зависимость (при различных длительностях падающего волнового пакета), расположение координат полюсов и седловых точек на комплексной плоскости интегрального преобразования Зоммерфельда-Ватсона для различных частот $x_{0}$, частотную зависимость для каждой моды поверхностной - волны (типа Рэлея, Стонелли, шепчущей галереи), полярную диаграмму направленности для различных мод при некоторых значениях частот. Совокупность таких характеристик позволит достаточно полно воссоздать картину рассеяния.

\section{Л И Т Е Р А Т У Р А}

1. Dragonette, L. R., F 1 ax, L., D ickey, J. W., Obera 11, H., In: 9 International congress on acoustics, Contributed papers, Madrid, 1977, p. L11.

2. Dickey, J. W., Ober a 11, H., Ibid., p. L12.

3. D i ckey, J. W., U beral1, H., J. Acoust. Soc. Amer., 63, № 2, 319-320 (1978).

4. Flax, L., Drag onette, L. R., Oberal1, H., J. Acoust. Soc. Amer,, 63, № 3, $723-731$ (1978).

5. D ickey, J. W., U ber a 11, H., J. Acoust. Soc. Amer., 66, № 1, 275-283 (1979).

6. Векслер Н., К ор с у ск ий В., Изв. АН ӘССР, Физ. Матем., 29, № 3, 296-305 (1980). 
N. VEKSLER

\section{ELASTSETELT SILINDRITELT HAJUVATE AKUSTILISTE LAINEPAKETTIDE PÖHJUSTATUD PINNALAINETE RESONANTSIST}

On analüüsitud elastselt täissilindrilt ideaalsesse kokkusurutavasse vedelikku hajuva lainevä1ja rõhu ajalist sõltuvust sondeerivate lainepakettide kestusest ning sagedusest. On esitatud põhiliste lainepakettide analüütiline kuju ja numbrilised karakteristikud ning hajunud rõhuvälja ajalised ja sageduslikud seosed. Silindri pinnal levivate Rayleigh' tüüpi elastsete pinnalainete tekitatud kiirgunud lainepaketid hakkavad resoneerima, kui pinnalainete lainepikkus mahub silindri perimeetrile täpselt täisarv kordi. Numbrilised tulemused vastavad juhule, kui terassilinder asetseb vees.

\section{N. VEKSLER}

\section{SURFACE WAVE RESONANCES IN SOUND PULSE SCATTERING BY ELASTIC CYLINDERS}

An analysis of the time function of sound pressure scattered by a solid circular elastic cylinder immersed in fluid medium is performed at short (Fig. 1) and long (Fig. 2) durations of the incident pulse and various values of the frequency. It is found that at the frequencies $x=5-25$ the main structure components of the scattered field pressure are the specular reflected pulse and the sequence of the re-radiated pulses caused by the Rayleigh type surface wave. Using either of the time functions (see Fig. 1, Fig. 2), one can determine the amplitude $A_{3 *}$ of the Rayleigh type surface wave at the moment of its excitation at the critical angle $\theta_{3}$ and the damping factor $\eta_{3}$. The correspondence between the time and frequency functions is shown: at $\tau \rightarrow \infty$ the average value of $p(\tau)$ tends to the value of $|p(x)|$ at $x=x_{0}$.

In the time domain the resonance nature of the interaction of the re-radiated wavepulses is shown. The resonance takes place when exactly an integer number wavelenghts of the surface wave fit the circumference of the cylinder. The numerical results are given in the case of an Armco iron cylinder immersed in water. 